\title{
Development of a dish-based food frequency questionnaire for Iranian population
}

\author{
Maryam Amini ${ }^{1}$, Ahmad Esmaillzadeh ${ }^{2}$, Nasrin Omidvar ${ }^{3}$, Mitra Abtahi ${ }^{1}$, Monireh Dadkhah Piraghaj ${ }^{1}$, \\ Bahareh Nikooyeh ${ }^{1}$, Tirang R. Neyestani*1 (D)
}

Received: 14 Apr 2019

Published: 30 Sep 2020

\section{Abstract}

Background: Food frequency questionnaires (FFQs) are widely used in large studies worldwide. They usually seek to rank people according to their usual food intake rather than a specific period of time. In the present study, we aimed to develop a dish-based, semiquantitative FFQ to seek habitual diet of general population aged 9 to 65 years in Tehran.

Methods: To develop the FFQ, four main steps were taken. At the beginning a list of commonly consumed Iranian foods and mixed dishes was prepared. To prevent excessive questionnaire length, some food items were grouped. Then, reference portion sizes were defined for each mixed dish in the list. The portion sizes were defined either based on the most common food portions reported in existing data or based on conventional portioning. In the third step the frequency response for consumption of food items and mixed dishes were determined. In the last step, recipes were developed for mixed dishes based on several data sources, including previous surveys. Finally, the content validity of the questionnaire was evaluated by the expert panel.

Results: The final food list of the FFQ comprised 142 food items and mixed dishes in six major food groups, including dairy products, breads, mixed dishes, dried fruits, nuts and seeds, fruits and vegetables, and miscellaneous food items and beverages. Nine frequency response options for all food items varying from "never or less than once per month" to "more than 6 times a day" were considered. A portion size was included for each item, whether food items or mixed dishes.

Conclusion: As Iranian food recipes are typically characterized by various ingredients it is very difficult for the respondent to recall the amount and type of the ingredients. Likewise, information about cooking methods beside ingredients of foods are important to estimate their nutritive values, which can be explored only through asking about consumption of mixed dishes. The current FFQ was developed to overcome the mentioned problems. It can be applied in nationwide studies in which foods and/or nutrients are predominant determinant of health and/or diseases.

Keywords: Questionnaire design, Community surveys, Epidemiology, FFQ

Conflicts of Interest: None declared

Funding: This work was supported by National Nutrition and Food Technology Research Institute, Islamic Republic of Iran (Grant no.450/1901-28/09/2016).

\section{*This work has been published under CC BY-NC-SA 1.0 license.}

Copyright $\odot$ Iran University of Medical Sciences

Cite this article as: Amini M, Esmaillzadeh A, Omidvar N, Abtahi M, Dadkhah Piraghaj M, Nikooyeh B, Neyestani TR. Development of a dish-based food frequency questionnaire for Iranian population. Med J Islam Repub Iran. 2020 (30 Sep);34:129. https://doi.org/10.47176/mjiri.34.129

\section{Introduction}

To assess nutritional status among individuals and communities, different methods of dietary assessment may be

Corresponding author: Dr Tirang R. Neyestani, t.neyestani@sbmu.ac.ir

1. Department of Nutrition Research, Faculty of Nutrition Sciences and Food Technology, National Nutrition and Food Technology Research Institute, Shahid Beheshti University of Medical Sciences, Tehran, Iran

2. Department of Community Nutrition, School of Nutritional Sciences and Dietetics, Tehran University of Medical Sciences, Tehran, Iran

3. Department of Community Nutrition, Faculty of Nutrition Sciences and Food Technology, National Nutrition and Food Technology Research Institute, Shahid Beheshti University of Medical Sciences, Tehran, Iran applied. Food frequency questionnaires (FFQs) have been widely used in epidemiological studies for several reasons.

$\uparrow$ What is "already known" in this topic:

Food frequency questionnaire, which are widely used in epidemiological studies, seek habitual dietary intake of people. They usually collect data based on food items rather than dishes, which may cause information bias.

\section{$\rightarrow$ What this article adds:}

This article can be used as a guide for other researchers to develop a dish-based food frequency questionnaire to reduce information bias regarding ingredients of foods and cooking methods. 
Compared to other methods of dietary assessment, FFQs are more feasible in community-based studies, because they impose minimal burden on respondents and are processed rather easily and inexpensively. Furthermore, they are cost-effective, time-efficient, and provide a reasonable estimate of the usual dietary intake over a long period (1, 2). Furthermore, FFQs are basically used in studies in which the precise amounts of consumed foods are less considered than ranking of people according to intake of nutrient(s) or food(s) of interest (1) .

Since dietary intake is influenced by many factors, such as culture, ethnicity, socioeconomic status, and individual's preference, FFQs are usually designed and developed based on the study group and research objectives. To our knowledge several FFQs have been developed and validated in Iran which are mainly based on food items alone and not mixed dishes (3-8).

Iranian food recipes are typically characterized by various ingredients prepared by different methods of cooking, thus making it very difficult for the respondent to recall the amount and type of the ingredients. On the contrary, in Western dishes food components are usually easily recognizable and determined at first glance for those not involved in cooking. On the other hand, it seems the consumption of ready to eat mixed dishes, either at home or in restaurants, has increased in recent years. Likewise, information about cooking methods in addition to ingredients of foods are important to estimate their nutritive values, which can be explored only through asking about the consumption of mixed dishes rather than food ingredients.

Since, two formerly developed dish-based FFQs in Iran were not validated, they were neither up-to-date nor in the scope and aim of our study; thus, we aimed to develop a new FFQ to overcome mentioned drawbacks $(9,10)$. In the present study, we aimed to develop a dish-based FFQ with multiple choice frequency response options to seek habitual diet of general population aged 9 to 65 years in Tehran.

\section{Methods}

To develop the FFQ, four main steps were taken (Fig. 1).

\section{Preparation of a food list}

At the beginning a list of commonly consumed Iranian foods and mixed dishes was prepared. TLGS (Tehran Lipid and Glucose Study) FFQ (8) was modified to make a list of primitive food items. TLGS FFQ included 168 food items and assessed the dietary intake of a person in the preceding 12 months. The list of mixed dishes was developed according to existing data, which included former national and local projects on dietary assessment (11-13).

Grouping of food items: To prevent excessive questionnaire length, some food items and mixed dishes were grouped. The grouped food items for nutritive value were similar.

Number of food items: First, a long list, including food items and mixed dishes was prepared. In regular meetings, the research team, consisting of a group of well experienced nutritionists selected food items and mixed dishes of the list, based on consumption data and making interindividual variations. The final list was reduced to 142 items (food items and mixed dishes).

Assembling the list of selected foods/dishes: The order of food items or mixed dishes in the questionnaire was arranged in the way to facilitate recalling and reporting of the items. We assumed it was easier for people to remember the consumed food based on order of eating meals during a typical day. The questionnaire was, therefore, initiated with food items that were commonly consumed at breakfast

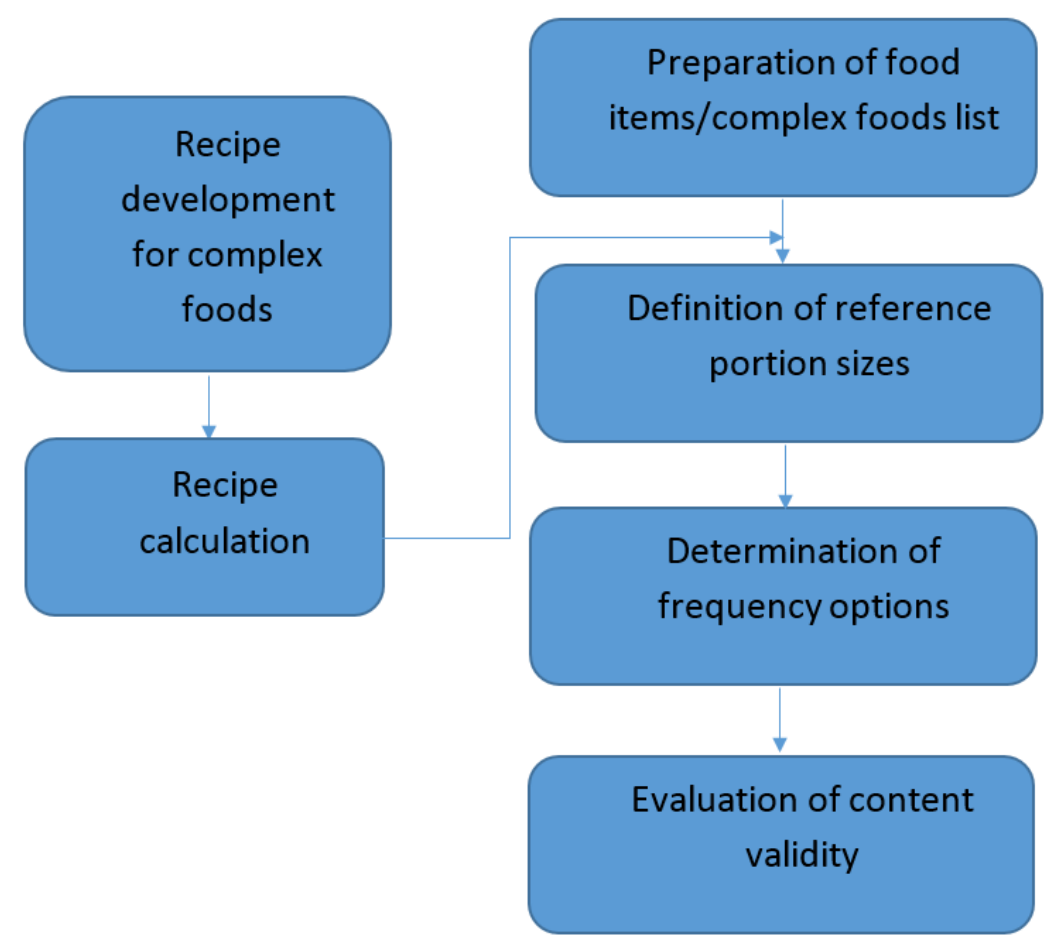

Fig. 1. Steps taken for development of the FFQ 
(such as dairy products and breads) and ended with snacks, which are more possibly to be eaten between main meals including fruits, sweets, crisps and etc.

\section{Recipe development for mixed foods}

Several data sources were obtained to develop a unique recipe for each mixed food (58 mixed dishes) (11-16). All data sources were searched to find all recipes for each mixed food. Ingredients of all related recipes were extracted and listed in a sheet (Table 1). As it is shown in Table 1, for each mixed food all ingredients of accessible recipes were recorded. List of all ingredients was extracted and the row "ingredients" included the overall ingredients in all available recipes. If a recipe lacked an ingredient it would be left blank. Number of recipes ranged between 2 to 30 . In the case that number of recipes were not enough (at least 2) other sources (e.g., cook books, cooking websites) were searched (14-16). If the number of recipes was still not enough or the collected information was not satisfying (for example, if the composition of recipes or amounts of recipes' ingredients were not logical), we searched other books than cook books (16) or interviewed chefs. Decision about odd measures (outliers) and overall combination and measures of recipes were based on a priori approach.

\section{Recipe calculation for mixed dishes}

Sum of energy and nutrient values of each recipe was calculated per portion size. We considered raw ingredients and included yield factor before calculation. Calculation was run by COMPUTE command in SPSS (Statistical Package for the Social Sciences) and yielded both amount of each food (gram) and its nutrients and energy value. The database of United States Department of Agriculture (USDA) was used to calculate energy and nutrients.

\section{Definition of reference portion sizes}

To calculate absolute nutrient intake, having information on portion sizes was crucial. As there are still no standard portion sizes for mixed dishes of different provinces of Iran, the research team discussed the portion sizes to suggest the best option for each food item. The portion sizes were defined either based on the most common food portions reported in the previous studies (17) or based on conventional portioning; for example, a bread portion equal to palm of a hand, a cheese portion equal to a matchbox, or a bowl of yogurt. Nonetheless, to finalize the usual portion sizes we let the interviewee report the portion sizes they actually consumed. Then, we recorded different types of reported portion sizes and measured frequencies of each, and the most frequent portion sizes were defined as final portion sizes.

\section{Determination of frequency options}

In the third step the frequency response for consumption of food items and mixed dishes were determined. We used the nine frequency response options used in the Nurses Cohort Study (1) for all food items varying from "never or less than once per month" to "more than 6 times a day" to estimate the frequency of different food intakes. We reckoned by the proposed options all possible frequency response options among interviewees would be met. They were indicated in the top of a column for all food items (Table 2).

\section{Content validity}

In the last step the content validity of the questionnaire was evaluated by the expert panel. The questionnaire was sent to 19 experienced experts. Along with the questionnaire they were asked for comments on the mixed dishes (complex foods) list, frequency options, and portion sizes of the FFQ. At the end they were asked to add any more

Table 1. The sheet used to extract ingredients of different recipes to develop a unique recipe for each mixed dish

\begin{tabular}{|c|c|c|c|c|c|c|c|c|}
\hline $\begin{array}{c}\text { Ingredients of } \\
\text { Khoresht Gheymeh* (Small bowl) }\end{array}$ & $\begin{array}{l}\text { Split } \\
\text { pea } \\
(\mathrm{g})\end{array}$ & $\begin{array}{l}\text { Meat } \\
(\mathrm{g})\end{array}$ & $\begin{array}{c}\text { Onion } \\
(\mathrm{g})\end{array}$ & $\begin{array}{l}\text { Potato } \\
(\mathrm{g})\end{array}$ & $\begin{array}{c}\text { Tomato paste } \\
\text { (g) }\end{array}$ & $\begin{array}{l}\text { Salt } \\
(\mathrm{g})\end{array}$ & $\begin{array}{l}\text { Oil } \\
(\mathrm{g})\end{array}$ & $\begin{array}{c}\text { Lemon powder } \\
\text { (g) }\end{array}$ \\
\hline Recipe 1 & $\mathrm{X}$ & $\mathrm{X}$ & $\mathrm{X}$ & $\mathrm{NE}$ & $\mathrm{X}$ & $\mathrm{X}$ & $\mathrm{X}$ & $\mathrm{NE}$ \\
\hline Recipe 2 & $\mathrm{X}$ & $\mathrm{NE}$ & $\mathrm{NE}$ & $\mathrm{X}$ & $\mathrm{X}$ & $\mathrm{X}$ & $\mathrm{NE}$ & $\mathrm{X}$ \\
\hline Recipe 3 & $\mathrm{X}$ & NE & $\mathrm{X}$ & $\mathrm{X}$ & $\mathrm{X}$ & $\mathrm{X}$ & $\mathrm{NE}$ & $\mathrm{X}$ \\
\hline Recipe $n$ & $\mathrm{X}$ & $\mathrm{X}$ & $\mathrm{NE}$ & NE & $\mathrm{X}$ & $\mathrm{X}$ & $\mathrm{X}$ & $\mathrm{X}$ \\
\hline \multirow[t]{2}{*}{ Mean } & $\Sigma X$ & $\Sigma X$ & $\Sigma X$ & $\Sigma X$ & $\Sigma X$ & $\Sigma X$ & $\Sigma X$ & $\Sigma X$ \\
\hline & $n$ & $n$ & $n$ & $n$ & $n$ & $n$ & $n$ & $n$ \\
\hline
\end{tabular}

*Khoresht Gheymeh is a typical Iranian dish.

NE: Not Exists.

$\mathrm{X}$ : Amount of the food ingredients (gram) in each recipe.

Table 2. Sample of the questionnaire. For each food/mixed dish item a portion size was considered. The frequency response options ranged from never to more than 6 times a day for each row.

\begin{tabular}{|c|c|c|c|c|c|c|c|c|c|}
\hline $\begin{array}{l}\text { Food } \\
\text { (portion size) }\end{array}$ & 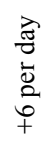 & 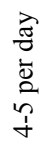 & 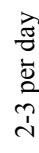 & 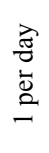 & b & 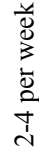 & 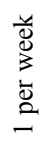 & 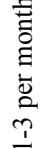 & 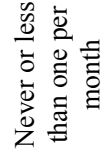 \\
\hline
\end{tabular}

\footnotetext{
Low fat milk (Cup)

Trditional bread/Lavash (A loaf)

Watermelon (Wedge)

Chelo (Plate)

Ghormesabzi (Bowl, small)

Soups (Bowl, medium)
} 
comments on the questionnaire. Almost $70 \%$ (68.4\%) of the panel commented on the FFQ. All point of views and the research team considered suggestions and finalized the questionnaire.

\section{Results}

The final food list of the FFQ comprised 142 food items and mixed dishes in six major food groups. The items of the food groups included (1) dairy products (dairies, butter and cream): 9 items; (2) breads (different types of breads, including traditional ones): 9 items; (3) mixed dishes: 58 items); (4) dried fruits, nuts, and seeds: 9 items; (5) fruits (and fruit juices) and vegetables (and vegetable juices): 25 items; and (6) miscellaneous food items and beverages (e.g., ice creams, sweets, synthetic juices, and carbonated beverages): 32 items. The questionnaire was initiated with milk and ended with salty snacks and included 9 frequency response options for each item. Regardless of being a food item or a mixed dish, all items of the FFQ included a portion size.

\section{Discussion}

To the best of our knowledge, at the time we intended to develop the current FFQ, two more dish-based questionnaires had been published in Iran. Initially, we thought of using the existing FFQs rather than developing a new one. The first dish based FFQ was developed and validated by Mashhad University of Medical Sciences (9). With respect to the validation of the questionnaire, we found only one published article. We preferred not to use Mashhad FFQ for two main reasons; first, the reported correlation coefficients of validation study were not acceptable; second, it was relatively old and needed to be updated. The second option was a FFQ, which was developed 10 years later for a study on the Epidemiology of Psychological, Alimentary Health and Nutrition (SEPAHAN) project (10). We were unable to use this questionnaire for two reasons. First, we found no publication indicating the validation of SEPAHAN FFQ. Second, the main objective of SEPHAN project was to seek any relationship between psychological and alimentary diseases with nutrition, which was different from our objectives. Hence, we decided to develop an FFQ de novo.

To prepare a food list in our FFQ, either the list of food items or the mixed dishes, we mainly depended on existing data, including previous national or local projects. Although sources of information, especially those from larger studies, were relatively old, we updated them with data of some recent studies. We faced a similar problem for recipe calculation. To overcome this challenge, we used other sources than information of studies. However, more information from large scale studies are needed to overcome the gaps.

Although steps taken for definition and finalization of foods' portion sizes in the present study are rigorous and expected to result in reliable information, fair judgment about their accuracy is possible after collecting data in the field and subsequent analyses.

To use the questionnaire for all Iran's climates and cul- tures, all mentioned steps should be done again to determine the recipe for all Iranian foods. There are some limitations to apply the questionnaire nationwide. First, because of the differences in various climates and access to food in Iran, this questionnaire cannot be used nationwide without further modifications. Second, the frequency options of the FFQ might not be the same for all foods. Some foods may be consumed much more frequently and some may not. Although our information about the frequency of consumption of all foods, especially mixed dishes, is not up-to-date and complete, the data which will be collected by this FFQ can provide valuable information in this regard.

Third, it may be argued that open-ended frequency options may yield more precise data. Nevertheless, it is documented multiple-choice frequency response options may result in more reliable data. First, they multiply clarity and thus reduce errors (18). Second, they are more feasible and practical for self-administered questionnaires; and third, for development of IT-based version of the FFQ, embedding close-ended rather than open-ended options is necessary.

Although the development of this questionnaire was an extensive task, we still have a long way to go before designing questionnaires for all climates and ethnicities in Iran. The current study paved the way toward the development of further questionnaires for more comprehensive nationwide studies. Furthermore, to be used in future studies the questionnaire initially needs to be validated against gold standards. These steps are being taken as a validation study whose results will be published in the near future.

\section{Conclusion}

Due to inclusion of mixed dishes, the newly developed FFQ is expected to assess dietary intake more precisely. The methodology explained here can be applied as a guide for the societies where consumption of mixed dishes is ordinary.

\section{Ethics approval and consent to participate}

The study protocol was approved by NNFTRI's ethics committee which follows the Helsinki Declaration (19). The ethics committee code was IR.SBMU.nnftri.Rec. 1395.43 .

\section{Acknowledgement}

We would like to express our gratitude to Dr. Azadeh Nikoosaleh for her contribution in development of recipes and Shahin Rajab for his contribution in FFQ development.

\section{Conflict of Interests}

The authors declare that they have no competing interests.

\section{References}

1. Willett W. Nutritional epidemiology, $2^{\text {nd }}$ edition. Oxford University Press; 2012 .

2. Shim J-S, Oh K, Kim HC. Dietary assessment methods in epidemiologic studies. Epidemiol Health. 2014;36.

3. Hosseini Esfahani F, Asghari G, Mirmiran P, Azizi F. Reproducibility and relative validity of food group intake in a food frequency 
questionnaire developed for the Tehran Lipid and Glucose Study. J Epidemiol. 2010;20(2):150-8.

4.Zeyninejad E, Omidvar N, Neyestani T, Houshiarrad A, Eshraghian MR, Stormer A. Development and Validation of a Food Frequency Questionnaire for Assessing Dietary Calcium in Children. Nutr Food Sci Res. 2015;2(1):35-45.

5. Dehghan M, López Jaramillo P, Dueñas R, Anaya LL, Garcia RG, Zhang X, et al. Development and Validation of a Quantitative Food Frequency Questionnaire among Rural- and Urban-dwelling Adults in Colombia. J Nutr Educ Behav. 2012;44(6):609-13.

6. Mohammadifard N, Omidvar N, Houshiarrad A, Neyestani T, Naderi G-A, Soleymani B. Validity and reproducibility of a food frequency questionnaire for assessment of fruit and vegetable intake in Iranian adults. J Res Med Sci. 2011;16(10):1286.

7. Mohammadifard N, Sajjadi F, Maghroun M, Alikhasi H, Nilforoushzadeh F, Sarrafzadegan N. Validation of a simplified food frequency questionnaire for the assessment of dietary habits in Iranian adults: Isfahan Healthy Heart Program, Iran. ARYA atherosclerosis. 2015;11(2): 139 .

8. Mirmiran P, Esfahani FH, Mehrabi Y, Hedayati M, Azizi F. Reliability and relative validity of an FFQ for nutrients in the Tehran Lipid and Glucose Study. Public Health Nutr. 2010;13(5):654-62.

9. Nematy M, Nouri M, Ghazizahedi S, Norouzy A, Mohajeri SAR, Shalaei N, et al. Validity and eproducibility of Iranian food frequency questionnaire. Nationalpark-Forschung in Der Schweiz (Switzerland Research Park Journal). 2014;102(12).

10. Keshteli AH, Esmaillzadeh A, Rajaie S, Askari G, Feinlel Bisset C, Adibi P. A Dish $?$ based Semi国quantitative Food Frequency Questionnaire for Assessment of Dietary Intakes in Epidemiologic Studies in Iran: Design and Development. Int $\mathrm{J}$ Prev Med. 2014;5(1):29-36.

11. Kalantari N, Ghaffarpour M. National Comprehensive Study on Household Food Consumption Pattern and Nutritional Status IR IRAN, 2001-2003. Tehran: Nutrition Research Department, National Nutrition and Food Technology Research Institute, Shaheed Beheshti University of Medical Sciences, Ministry of Health; 2005.

12. Amini M, Dadkhah-Piraghaj M, Abtahi M, Abdollahi M, Houshiarrad A, Kimiagar M. Nutritional assessment for primary school children in Tehran: An evaluation of dietary pattern with emphasis on snacks and meals consumption. Int J Prev Med. 2014;5:611-6.

13. Amini M. Developing and evaluating of an interventional model to prevent and control childhood obesity in primary school children of district 9 of Tehran. Thesis of the degree of Doctor of Philosophy in Nutritioal Sciences. Tehran University of Medical Sciences; 2014.

14. Montazemi R. Art of cooking. 1 ed: Ketabe Iran; 2012:943.

15. SanazSania 2016 [cited 2016]. Available from: http://www.sanazsania.ir/.

16. Ayatollahmadadi S, Bozorgmehr B. Therapeutic diet: Markaze Nashre Daneshgahi; 1989.

17. Ghaffarpour M, Houshiar-Rad A, Kianfar H, Banieghbal B. Food album: National Nutrition \& Food Technology Research Institute; 1997.

18. Ferreira MG, Silva NF, Schmidt FD, Silva RM, Sichieri R, Guimarães LV, et al. Development of a Food Frequency Questionnaire for adults in a population-based sample in Cuiabá, Mid-Western Region of Brazil. Rev Bras Epidemiol. 2010;13(3):413-24.

19. Association WM. World Medical Association Declaration of Helsinki, Ethical principles for medical research involving human subjects. Bullet World Health Organ. 2001;79:373]4. 